\title{
ADVANCES IN DIAGNOSIS AND DETECTION OF ORAL DISEASES
}

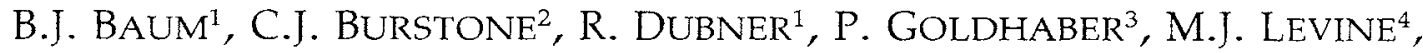 \\ W.J. LOESCHE ${ }^{5}$, and V. TERRANOVA ${ }^{4}$ \\ National Institute of Dental Research, National Institutes of Health, Bethesda, Maryland; ${ }^{2}$ University \\ of Connecticut, School of Dental Medicine, Farmington, Connecticut; ${ }^{3}$ Harvard University School of \\ Dental Medicine, Boston, Massachusetts; ${ }^{4}$ SUNY at Buffalo, School of Dentistry, Buffalo, New York; \\ and "University of Michigan, School of Dentistry, Ann Arbor, Michigan
}

Adv Dent Res 3(1):7-13, May, 1989

\begin{abstract}
$\mathbf{M}$ edicine, particularly with respect to diagnostic decision-making, has seen remarkable advances in the last ten years. The art of diagnosis has become much more of a science. Basic science advances have moved from the laboratory into the hospital and radically changed the way a medical diagnosis is arrived at or confirmed. Dentistry, especially oral diagnosis, as yet has not been a significant part of this general medical advance. However, several examples demonstrate that this situation is starting to change. Oral conditions are beginning to be evaluated with greater precision and sophistication. This report reviews some recent advances in oral diagnostic research and suggests where they will carry dentistry over the next 25 years.
\end{abstract}

\section{INTRODUCTION}

Medicine, particularly with respect to diagnostic decision-making, has seen truly remarkable advances in the last ten years. The art of diagnosis has become much more of a science. Technological advances from biochemistry, immunology, physics, etc., have moved from their laboratories into the hospital and combined to radically change the way a medical diagnosis is arrived at or confirmed.

Dentistry, especially oral diagnosis, as yet has not been a significant part of this general medical advance. But there are many examples, some of which are cited in the sections below, which demonstrate that this is beginning to change. Oral conditions are beginning to be evaluated with considerably greater sophistication. It is the role of this paper to suggest where these advances in oral diagnostic research and application will carry the diagnostic side of the profession over the next 25 years.

At the outset, it is worth stating that the authors recognize the precarious task of predicting the future,

A report of the AADR ad hoc Committee on New Frontiers in Oral Health Research

Please address all correspondence to Dr. Bruce J. Baum, Clinical Investigations and Patient Care Branch, National Institute of Dental Research, National Institutes of Health, Building 10, Room 1A05, Bethe'sda, MD 20892. particularly as it relates to biomedical science. For example, six years ago it would have been impossible to predict the profound and pervasive impact that AIDS would have on the biomedical research community. Yet this "epidemic" has markedly influenced the directions and nature of biologic research in 1988. Obviously, our level of confidence decreases with time, but we generally have accepted the notion that oral diagnosis as a discipline will change dramatically over the time frame considered. This change likely will reflect a much broader level of stomatological concern by future dentists than exists at present. In fact, as diagnostic skills and methodologies become more sophisticated, we believe that much professional oral diagnosis will be performed outside of the traditional dental office setting, occurring rather in a hospitaltype environment, and indeed being performed by an expanded specialty of more intensively trained oral diagnosticians. Just as dental research has contributed, and will continue to contribute, toward shifts in the nature of dental practice, research advances in oral diagnosis will contribute not only to changes in patient evaluation and management, but also to shifting emphases in dental education. Future students will require greatly expanded training in many basic and clinical medical science disciplines in order to utilize the anticipated diagnostic advances. Indeed, for the transfer to the clinical profession of the "advances" described in this paper, significant changes 
in dental education and clinical practice behavior will have to occur.

For purposes here, we have grouped anticipated advances into short-term ( $\sim 5$ years), intermediate $(\sim 10$ years), and long-term ( $\sim 25$ years) categories. We hope these provide a convenient time-line for the reader, but we emphasize that the time course is most speculative. Also, the number of predictions decreases with increasing time, reflecting what we feel is a greater confidence in our abilities to predict shorter-term trends.

\section{SHORT-TERM ADVANCES ( $~ 5$ YEARS)}

Research advances here focus on the transfer of methods and ideas, which are currently available in other biomedical areas, to oral diagnostic problems. In general, these examples were not hard to choose; in fact, there were many possibilities. However, all topics selected have by now brought great benefit to several areas of clinical medical science and, importantly, these topics have shown themselves to be clearly applicable to problems of the oral cavity. It is important to emphasize that these are diagnostic tools which are capable of being used now, the limitation being only problems in transfer to, or acceptance by, the profession.

\section{(1) Radionuclides in the Diagnosis of Periodontal Disease}

Nuclear medicine as a medical specialty has grown enormously in the last dozen or so years. Many radionuclides are in common clinical diagnostic usage, with more sophisticated probes being developed at an increasingly more rapid pace. The general principle involved is quite simple: The diagnostician administers a radiolabeled compound which shows particular affinity for a tissue and which reflects something about the metabolic or functional state of the tissue. Thereafter, some type of detector is used to quantitate the relative uptake of tracer in the tissue of interest. An excellent and common example of radionuclide diagnostic testing is the use of ${ }^{131} I$ or ${ }^{125} I$ in evaluations of thyroid gland metabolic status.

Recently, workers at the Harvard School of Dental Medicine have suggested the utilization of a radionuclide, technetium 99m-tin-diphosphonate (99mTcSn-MDP), as an indicator of active alveolar bone loss in the diagnosis of periodontal disease activity (Jeffcoat et al., 1985). Commonly used methods of detecting periodontal diseases require repeated measurements over considerable time to assess rate of attachment loss (i.e., periodontal probing). Similarly, radiographic analyses show only post hoc destruction of alveolar bone. Presently, there is considerable effort focused on methods of diagnosing active periodontal disease (Fine and Mandel, 1986). Jeffcoat and her colleagues recognized that $99 \mathrm{mTc}$ $\mathrm{Sn}-\mathrm{MDP}$ was in routine medical use to detect bony metastases, stress fractures, infections, etc., prior to the presence of radiographic evidence. Accordingly, they reasoned that uptake of this bone-seeking radionuclide might provide a diagnostic mechanism to assess periodontal status in a single examination. Their results, found in the report cited above, and summarized in Fig. 1, offer strong support for the utility of $99 \mathrm{mTc}-\mathrm{Sn}-\mathrm{MDP}$ in the diagnosis of periodontal disease.

The possibilities for application of nuclear medical technology to oral problems seem to be limitless, requiring only imagination, a good knowledge of tissue physiology, and a cooperative chemist who can design a radiolabeled ligand with appropriate target specificities. There are several radionuclides already in general medical use which should soon find their way into oral diagnosis - another good example being ${ }^{99} \mathrm{mCO}_{4}{ }^{-}$for evaluating salivary gland dysfunction. We expect there will be considerable investigative effort in this area over the next 5-10 years, resulting in a vastly more sophisticated armamentarium for oral diagnosticians.

\section{(2) Direct Imaging Methods}

Another major tool in medical diagnosis which has yet to find its way into common oral diagnosis is the utilization of advanced direct imaging methods. The clinical specialty of radiology has been expanded widely due to the rapid development and application of a new generation of imaging devices. It would be easy to devote this entire short-term advances section

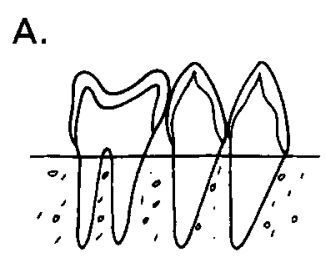

B.

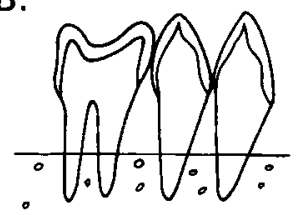

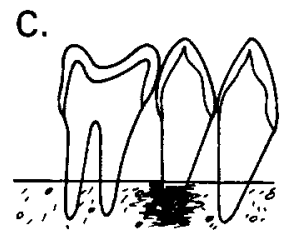

Fig. 1-Schematic representation of detection of active periodontal disease with $99 \mathrm{~m}$-tin-diphosphonate. A. Normal alveolar bone appearance observed with little bone-seeking radiopharmaceutical uptake (black flecks) associated with the bone about the teeth. B. Representation of area with evidence of past periodontal disease. There is horizontal bone loss about the teeth but no radiopharmaceutical uptake, indicating no active disease. C. Area similar to that shown in B, but note that the middle tooth shows an adjacent area of bone which has taken up large amounts of the $99 \mathrm{~m}$-tindiphosphonate tracer, indicating an area of active bone loss. This area may be detected by means of a small gamma emission detector placed on the patient's gingiva. 
to these technologies. Indeed, as further indicated below, we expect later advances in diagnosis to be particularly directed at more sophisticated physical science probes.

As with radionuclides, we are best able to show the value of such advanced imaging methods to oral diagnosis through an example of recent applications. Considerable investigative effort, with an oral problem area, has come from the use of ultrasound to detect swallowing disorders (dysphagia) of oral etiology. In principle, ultrasonography functions similarly to sonar on a submarine - i.e., transmitting a sound wave and, by detecting reflections of sound off objects in its path, "visualizing" structures. In this case, ultrasound waves are passed through tissues, and reflections of these waves are converted to an image by appropriate hardware. Ultrasound is an excellent means of visualizing soft tissues in active function (e.g., echocardiography).

Workers at the National Institutes of Health have utilized this technology to evaluate and characterize swallowing disorders in the oropharyngeal region (Shawker et al., 1983). The dorsum of the tongue:air interface offers virtually perfect impedance to an ultrasound wave, resulting in a sharp visualization of the tongue during function (Fig. 2). Swallows using endogenous oral secretions ("dry" swallows) or those with a bolus of fluid present (e.g., water; "wet" swallows) can be followed in real time, recorded on video
A.

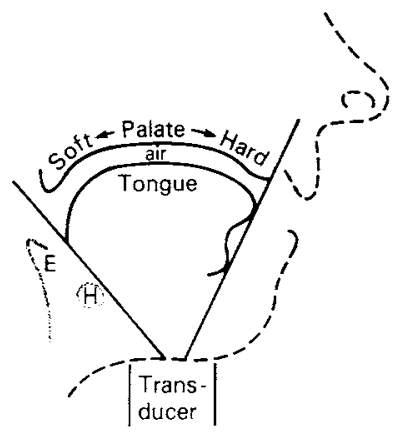

B.

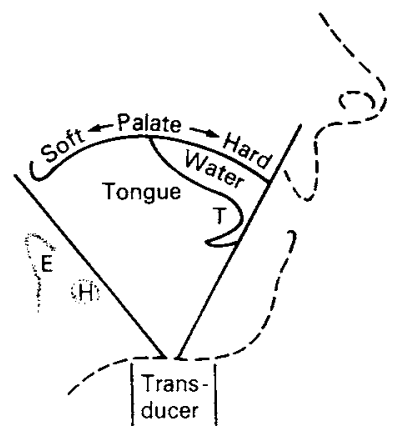

Fig. 2-Schematic diagram of midline sagittal views of the tongue and floor of the mouth as seen on ultrasound scan. The area encompassed by the scan is indicated by the somewhat truncated "V-shape" ( $\sim 80^{\circ}$ sector). The ultrasound transducer is positioned under the chin. Dashed lines indicate structures not seen in scan and are placed here only for reference. $A$. The tongue at rest. There is a small air space between the tongue and palate. B. A water bolus ( $5 \mathrm{~mL}$ ) has been placed in the mouth and is held anteriorly, anticipating a command to swallow. The air space above the posterior tongue is no longer visible. Abbreviations used are $T$, tongue tip; $H$, hyoid bone; $E$, epiglottis. Swallow analysis can be performed with only endogenous secretions present (i.e., saliva, as depicted in A, termed "dry swallow") or with an exogenous fluid bolus (i.e, water, depicted in B, termed "wet swallow"). Tongue movement is recorded on videotape at a rate of 30 frames/sec and can be analyzed by stop-frame analysis. tape, and analyzed for time kinetics, pattern of motion, irregular gestures, etc. This is a non-invasive, readily repeatable technique which allows for imaging of oral functions and tissues heretofore impossible with conventional radiologic tools. In actuality, little study of oropharyngeal dysphagia has occurred, yet it appears to be a reasonably common entity (Hughes et al., 1987). For example, patients with hypofunctional salivary glands, regardless of etiology (e.g., radiation, autoimmune disease, pharmacological), routinely display, and complain of, swallowing difficulty. Also, many patients, post-treatment for head and neck tumors, may suffer from a dysphagia of oropharyngeal etiology.

There are other imaging tools (computer-assisted tomographic analysis; magnetic resonance) which are in increasingly more common use in medical diagnosis and which have also been applied to oral problems, e.g., temporomandibular joint disorders (Skaleric et al., 1987). They have potentially broad applications to oral tissues. We certainly expect this general field to contribute considerably to advances in oral diagnosis over the next 5-10 years.

\section{(3) Humoral Markers for Pain and Tissue Injury}

Recent advances in the understanding of the biochemistry and pharmacology of pain pathways have led to the use of chemical intermediates as markers of both pain and tissue injury. Currently used methods to analyze these markers in conjunction with management decisions for pain patients are an excellent example of how humoral analyses may be employed or adapted to problems in oral diagnosis. For instance, pain produces physiological responses involving activation of the hypothalamic-pituitary-adrenal axis and the consequent secretion of neurohumoral agents from these tissues. Thus, corticotropin-releasing factor is released from the hypothalamus; adrenocorticotrophic hormone, betaendorphin, and beta-lipotropin are released from the pituitary; and the adrenal medulla releases monoamines and opiate peptides. All of these humoral agents can be easily measured via standard immunochemical methods (radioimmunoassay) and correlated with behavioral measures of pain and stress (Fig. 3). Recent studies have shown that opiate agonists given during surgery suppress beta-endorphin release and reduce pain, while opiate antagonists stimulate the release of beta-endorphins and are associated with increased pain (Hargreaves et al., 1986). Thus, the levels of opioid peptides in plasma seem to be useful diagnostic markers for quantitating the amount of pain and stress perceived by patients. Similar analyses can be performed for norepinephrine and serotonin.

A comparable monitoring of another area of oral diagnostic concern, tissue injury (trauma, post-surgery), can be achieved by following plasma levels of substances such as prostaglandins, leukotrienes, bradykinin, and substance P. All of these analyses also 


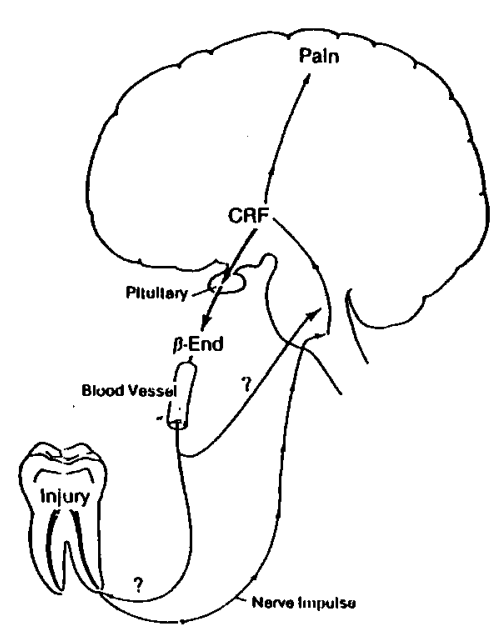

Fig. 3-Schematic representation of the role of humoral mediators in pain relief. An injury to the tooth sends a nerve impulse to the brain causing release of CRF (corticotropin-releasing factor) from the hypothalamus. CRF next signals the pituitary to secrete Bendorphins ( $\beta$-End) in response to this pain stress. $\beta$-Endorphins circulate through the blood stream and may act to relieve pain either at the site of injury (the tooth), or in the brain. $\beta$-Endorphins can be easily measured in blood samples taken from patients by a radioimmunoassay procedure and can then be used as a "physiological" measure to compare with behavioral measures of pain made in parallel.

utilize radioimmunoassays with specific antibodies. These diagnostic tests allow for accurate staging of clinical problems and therefore for accurate, and more specific, therapeutic intervention.

Development of other humoral analyses which correlate with oral health status will likely be the subject of considerable investigative effort over the next few years. The cited, currently available examples clearly attest to the increased accuracy, specificity, and sensitivity of such procedures as well as to their applicability and usefulness with respect to problems faced by the oral diagnostician.

\section{INTERMEDIATE ADVANCES ( 10 YEARS)}

Research advances here focus on the development of new diagnostic tools/methodologies, which are not universally available to clinical medicine and dentistry, but which at present exist conceptually, and practically, in the laboratory setting. These predictions are derived from advances at the "current edge" of modern biology.

\section{(1) Molecular Probes for Identifying Tumor Cells and Identifying Specific Therapies}

There has been a veritable explosion of knowledge in the last few years about how cell growth is con- trolled. The focus of this development can arguably be assigned to cellular oncogenes, eukaryotic cellular genes which have considerable homology to oncogenes from transforming viruses. Several categories of cellular oncogenes, and their phenotypic protein products, have been identified, and nucleotide probes are available to detect their presence in cells. At present, many laboratory studies are directed toward identifying oncogene expression or amplification following developmental or mitogenic stimuli. However, a few studies have identified the presence of amplified oncogenes in human malignancies. For example, Aaronson and his colleagues at the National Cancer Institute have demonstrated amplification of the mac oncogene in $\sim 15 \%$ of human mammary carcinomas (King et al., 1985). Similarly, a research group in Japan has identified oncogene amplification in a salivary gland carcinoma (Semba et al., 1985). These malignant cells may be thought to display their uncontrolled growth due to this amplification process. The specific derepression event(s) allowing for gene amplification is at present not known, but we can anticipate progress toward its identification within five or so years. Thus, we can predict that in 10 years, for some malignant tumors, we will know the gene derangement which results in uncontrolled cell growth, including the actual perturbed, permissive genetic switch. Similarly, we will know, and be able to identify (immunologically), the protein products of these oncogenes.

Accordingly, we expect that oral diagnostic research related to cancer will in part be directed at identifying these genetic signals. This will allow future diagnosticians to use these types of probes routinely to identify the specific etiology of a malignant tumor, both by molecular hybridization studies with biopsy samples and by analyses of tissue or serum for protein products of malignant transformation. In principle, these techniques are quite simple (Fig. 4). For example, nucleotide probes can interact specifically (hybridize) with complementary nucleic acid sequences in cells in situ or in extracted DNA. If the oligonucleotide probe is labeled (e.g., isotopically), it can be detected readily, thereby indicating the presence of the gene sequence of interest. The protein products of these genes can be easily identified by immunoassays, as noted earlier. With such precise diagnostic knowledge, it will also be possible to design exact therapeutic reagents for specific types of tumors. These can be targeted only to cells expressing the malignant aberration - a considerable advance over current non-specific forms of chemotherapy which affect normal as well as tumor cells. These advances, for general medicine, are now not far from achieving routine practicality. Indeed, the techniques described in Fig. 4 have been applied to identify viruses in various tissues and have been useful in suggesting the viral etiology of a specific oral AIDS-associated lesion (e.g., Greenspan et al., 1985). 
A.

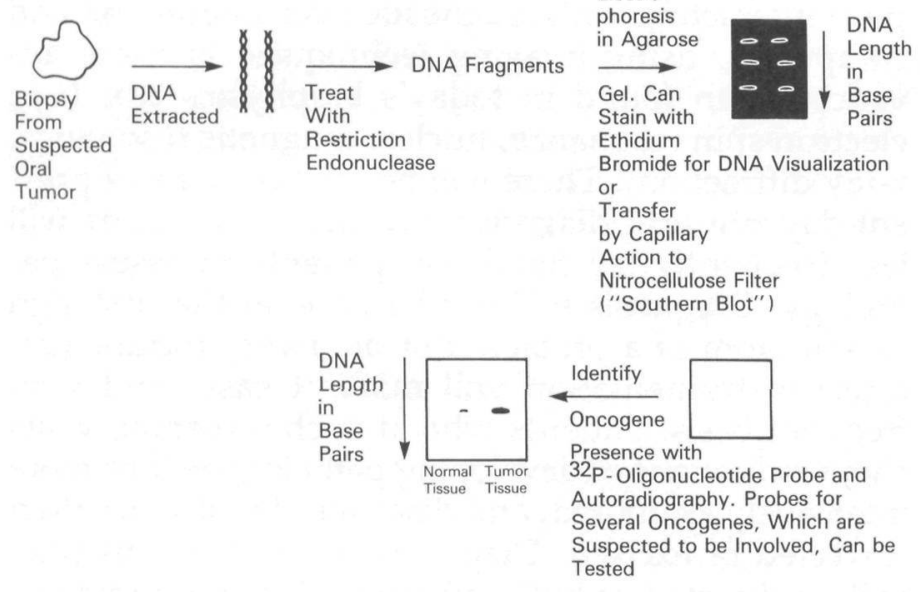

B.
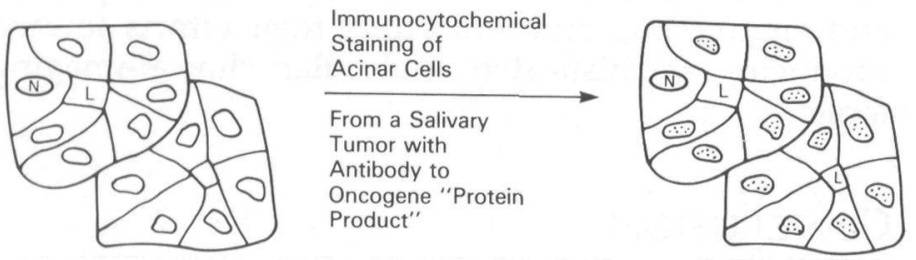

Fig. 4-Diagnostic methods to identify oncogenes in a biopsy from a patient's tumor. A. Using molecular biological/biochemical procedures. B. Using immunocytochemical procedures. In Fig. 4B, the biopsy is fixed and incubated with buffer containing antibody to the oncogene "protein product". Next, the tissue is washed, and a second antibody, coupled to peroxidase, is added. After another wash, substrates for peroxidase are added. Deposited reaction product is seen (black dots) where the oncogene "protein product" is localized (here, in nucleus).

\section{(2) Diagnostic Probes for Assessing Functional Capacity of Salivary Epithelial Cells}

In the last two to four years, our knowledge about how saliva formation takes place has expanded enormously. We have recognized several of the ion channels and carrier-mediated transporters which are necessary for transepithelial cell water movement. These include $\mathrm{Ca}^{2+}$-activated $\mathrm{K}^{+}$channels, $\mathrm{Ca}^{2+}$-activated $\mathrm{Cl}^{-}$channels, and the $\mathrm{Na}^{+} / \mathrm{K}^{+} / \mathrm{Cl}^{-}$co-transporter. We also know most of the neurotransmitter receptor control mechanisms involved in these fluidgenerating events. Current laboratory developments in identifying receptors by experimental imaging methods and the advent of isotopic probes for some ion transport proteins (particularly the $\mathrm{Na}^{+} / \mathrm{K}^{+} / \mathrm{Cl}^{-}$ co-transporter) suggest that research efforts in $\sim 10$ years hence will be directed at devising methods to allow oral diagnosticians to ask discrete questions about the status of an ion channel, a transport protein, or a receptor coupling mechanism.

Salivary gland dysfunction is a reasonably common occurrence. Autoimmune exocrinopathies affect from two to four million Americans, and iatrogenic causes of gland dysfunction (radiation, pharmaceuticals) are frequently seen. In the extreme case (e.g., destruction due to autoimmune disease or to radiation damage), gland dysfunction may be, at present, reasonably straightforward to diagnose. However, there are many disorders of salivary glands for which a definitive diagnosis is difficult to reach. Obviously, management of these diagnostically elusive situations is also inadequate. There are now several examples in medicine of diseases which result from subtle disturbances in receptors or transduction molecules. The bestcharacterized of such deficits is pseudohypoparathyroidism (Van Dop and Bourne, 1983). It is highly likely that there are salivary (and other oral tissue) disorders which result from such discrete molecular disturbances. Future research will take the current generation of available molecular and immunologic probes to more practical levels (e.g., radionuclides coupled to probes; Drayer et al., 1982), making routine assessments of the status of key acinar cell plasma membrane proteins possible both in situ, during actual function (Fig. 5), as well as with biopsied material in vitro.

Such diagnostic progress, coupled with the present progress with in vitro eukaryotic cell gene transfection procedures and the anticipated success of gene transfection techniques in humans, will pave the way for long-term future research advances in managing salivary disorders. For example, we can speculate that such research will eventually lead to the following, or a similar, clinical management scenario. After determining that a patient is unable to secrete saliva because acinar cells possess an apical membrane $\mathrm{Cl}$ channel which is defective and unresponsive to stimuli, future clinicians will be able to target the insertion of the gene coding for a functional $\mathrm{Cl}^{-}$channel, or a modifier molecule, via cell-directed vectors and thus restore gland function (Wang and Huang, 1987).

The intermediate period covered here will be one that will make commonplace the transfer of today's laboratory molecular biological advances into the routine clinical setting. Technically, it is becoming increasingly easier to produce nucleotide, peptide, or immunologic probes of exquisite specificity. The oral diagnostician of the not-too-distant future will indeed make molecular diagnoses. We feel very confident that at the period around the turn of this century, oral diagnostic research will see such tools in conventional use for a variety of oral problems such as neoplasia and non-neoplastic oral mucosal diseases, viral infections, autoimmune disorders, and gustatory and other sensory dysfunctions.

\section{LONG-TERM ADVANCES ( 25 YEARS)}

It would not be surprising that by this time, in the economically advanced countries of the world, traditional oral diseases (caries, periodontal diseases) will not be significant clinical problems. Simple kits, to use at home, will be available for general monitoring 


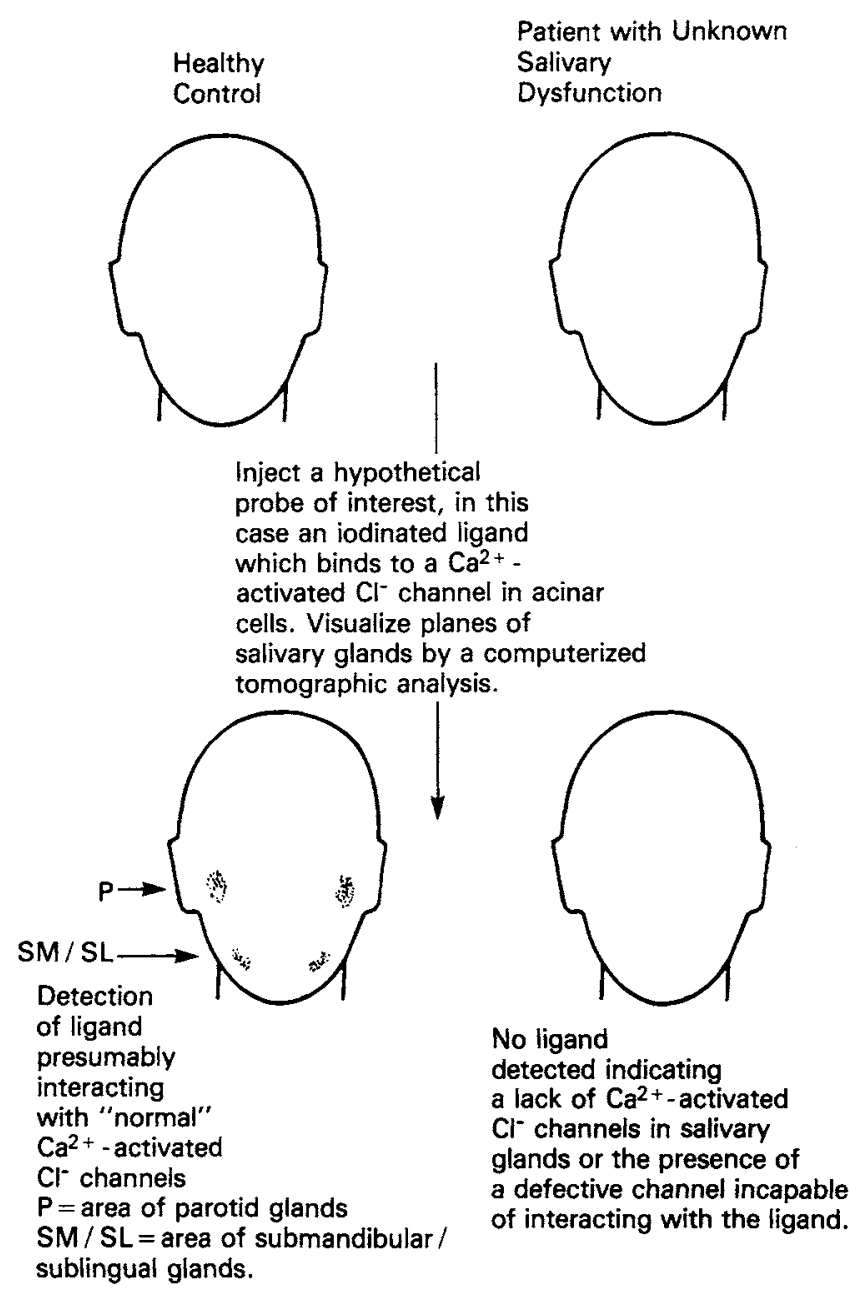

Fig. 5-Use of a radionuclide-coupled probe, in conjunction with photon emission computed tomography, to assess the functional status of salivary acinar cell $\mathrm{Cl}^{-}$channels.

of dental disease, salivary gland function, oral infections, etc. In addition, it is possible that most forms of cancer will be easily diagnosed and readily treated. There will still be a place for biologically oriented diagnoses within dentistry, although we speculate that many "oral" diagnostic decisions will be in the hands of a relatively small group of highly trained individuals $(\sim 5000)$ who will practice in sophisticated diagnostic centers and concentrate on what now might be thought of as subtle glandular, neural, muscular, and skeletal disorders of the head, neck, and face. Research emphasis in diagnosis will be substantially different from that seen today; physical science will dominate over biological science. Phenomenal general technological advances will be made in the aerospace, communications, and defense industries over the next 20-30 years, with spin-offs to all facets of daily life. This will lead to revolutionary applications in physiology and health sciences. In many ways, to us now, these belong in the realm of science fiction.
We expect to see many patients (and patient samples) interacting with diagnostic instrumentation and computers, using imaging techniques far more advanced than found in today's biophysics labs (i.e., electron spin resonance, nuclear magnetic resonance, $x$-ray diffraction). There will be little or none of present-day physical diagnosis practiced. Clinicians will less frequently lay hands on patients to assess pathology. Diagnosis will not be done at the first sign or symptom of a problem but on a very routine (because instrumentation will make it easy) and very frequent basis. Patients who at such screening visits show indications of developing pathology will be more intensively examined, and disordered molecules then corrected or excised. Diagnosis research at this time will be directed at both submolecular and supramolecular levels. Individuals involved will have biological backgrounds but will be heavily trained in physics and engineering and will direct their efforts toward producing sophisticated molecular clinical-imaging tools.

\section{CONCLUSION}

All of medicine will change dramatically over the next 25 years, perhaps diagnosis more than most areas. The changes will be fueled by advances in other, nonrelated, fields, but also by the elimination of many modern diseases. For comparison, take the case of the medical diagnostician who graduated in 1940 before World War II and only retired last year from practice. He (she) has witnessed a dramatic shift in the diseases he was called upon to detect, the means with which he could detect them, and the tools with which he could treat them. Similarly, the oral diagnostician will be shifted in focus dramatically in the next 10-25 years. By the turn of this century, advances in oral diagnosis will be a full partner of mainstream medical advances.

It is, as noted earlier, hard for us to predict with any reasonable level of accuracy advances beyond a $\sim 10$-year time-point. We all, however, recognize that advances are occurring at exponential rates. Most importantly, we believe that the next 10 years or so are a time of almost unrestricted opportunity in oral diagnosis research. It is a time of new directions, which can be taken readily from guidelines offered through current advances in biology and medicine. We believe that this should give considerable encouragement both to young oral health investigators concerned about future clinical research opportunities and to the dental profession at large, because new practice vistas will be charted. Since many of these advances are in areas which are non-traditional with respect to dental practice, we believe that substantial attention must be paid to transferring advances to the clinical arm of the profession. This portends significant changes in dental education directed both at the ac- 
quisition of the necessary scientific background and the alteration of existing "practice behaviors".

\section{REFERENCES}

DRAYER, B.; JASZCZAK, R.; COLEMAN, E.; STORNI, A.; GREER, K.; PETRY, N.; LISCHKO, M.; and FLANAGAN, S. (1982): Muscarinic Cholinergic Receptor Binding: in vivo Depiction Using Single Photon Emission Computed Tomography and Radioiodinated Quinuclidinyl Benzilate, J Computer Assist Tomogr 6: 536-543.

FINE, D.H. and MANDEL, I.D. (1986): Indicators of Periodontal Disease Activity: an Evaluation, J Clin Periodontol 13: 533-546.

GREENSPAN, J.S.; GREENSPAN, D.; LANNETTE, E.T.; ABRAMS, D.I.; CONANT, M.A.; PETERSEN, V.; and FREESE, U.K. (1985): Replication of Epstein-Barr Virus within the Epithelial Cells of Oral Hairy Leukoplakia, an AIDS-associated Lesion, $N$ Engl I Med 313: 1564-1571.

HARGREAVES, K.M.; DIONNE, R.A.; MUELLER, G.P.; GOLDSTEIN, D.S.; and DUBNER, R. (1986): Naloxone, Fentanyl and Diazepam Modify Plasma Beta-Endorphin Levels During Surgery, Ciln Pharmacol Ther 40: 165-171.

HUGHES, C.V.; BAUM, B.J.; FOX, P.C.; MARMARY, Y.; YEH, C.-K.; and SONIES, B.C. (1987): Oral-Pharyngeal Dysphagia: a Common Sequela of Salivary Gland Dysfunction, Dysphagin 1: $173-177$.
JEFFCOAT, M.K.; WILLIAMS, R.C.; KAPLAN, M.L.; and GOLDHABER, P. (1985): Bone-seeking Radiopharmaceutical Uptake as an Indicator of Active Alveolar Bone Loss in Untreated and Surgically Treated Teeth in Beagle Dogs, I Periodont Res 20: 301306.

KING, C.R.; KRAUS, M.H.; and AARONSON, S.A. (1985): Amplification of a Novel v-erb B-related Gene in a Human Mammary Carcinoma, Science 229: 974-976.

SEMBA, K.; KAMATA, N.; TOYOSHIMA, K.; and YAMAMOTO, T. (1985): A v-erb B-related Protooncogene, c-erb B-2, is Distinct from the c-erb B-1/Epidermal Growth Factor-Receptor Gene and is Amplified in a Human Salivary Gland Adenocarcinoma, Proc Natl Acad Sci USA 82: 6497-6501.

SHAWKER, T.H.; SONIES, B.; STONE, M.; and BAUM, B.J. (1983): Real-time Ultrasound Visualization of Tongue Movement During Swallowing, I Clin Lltrasound 11: 485-490.

SKALERIČ, U.; DOLINŠEK, J.; STEPIŠNIK, J.; CEVC, P.; and SCHARA, M. (1987): NMR Imaging in Dentistry: Relaxation and Diffusion Studies, Adv Dent Res 1: 85-87.

VAN DOP, C. and BOURNE, H.R. (1983): Pseudohypoparathyroidism, Ann Rev Med 34: 259-266.

WANG, C.-Y. and HUANG, L. (1987): pH Sensitive Immunoliposomes Mediate Target-cell-specific Delivery and Controlled Expression of a Foreign Gene in Mouse, Proc Natl Acad Sci USA. 84: $7851-7855$ 